\title{
Kernos
}

Revue internationale et pluridisciplinaire de religion grecque antique

12 | 1999

Varia

\section{J.M. BLÁZQUEZ, Intelectuales, ascetas y demonios al final de la Antigüedad}

\section{Pier Franco Beatrice}

URL : http://journals.openedition.org/kernos/742

DOI : $10.4000 /$ kernos.742

ISSN : 2034-7871

\section{Éditeur}

Centre international d'étude de la religion grecque antique

\section{Édition imprimée}

Date de publication : 1 janvier 1999

Pagination : 317-318

ISSN : 0776-3824

Référence électronique

Pier Franco Beatrice, « J.M. BLÁzauez, Intelectuales, ascetas y demonios al final de la Antigüedad », Kernos [En ligne], 12 | 1999, mis en ligne le 13 avril 2011, consulté le 11 mars 2021. URL : http:// journals.openedition.org/kernos/742 ; DOI : https://doi.org/10.4000/kernos.742 
José María BLÁzQuez, Intelectuales, ascetas y demonios al final de la Antigüedad, Madrid, Ediciones Cátedra, 1998. 1 vol. $13,5 \times 21 \mathrm{~cm}, 566$ p. ISBN : 84-376-1499-6.

Il s'agit d'un recueil d'essais de l'illustre savant espagnol, déjà publiés au cours des dernières années dans différents livres, revues et mélanges, et qui sont maintenant réunis et réédités, avec les développements et les mises à jour appropriés, dans ce beau volume qui en simplifie la consultation, surtout pour des lecteurs qui ne peuvent pas accéder facilement aux publications espagnoles.

Bien que traitant de différents arguments, les essais qui sont recueillis ici ont en commun la base documentaire car ils examinent, tous sans exception, les écrits des auteurs chrétiens des premiers siècles. Le volume prouve donc d'abord la grande familiarité de l'auteur avec la littérature patristique grecque et latine. Il faut signaler cet aspect avec une vive approbation car il est assez rare que les historiens de l'antiquité s'appuient sur les sources chrétiennes de la fin de l'antiquité comme c'est le cas dans ces pages denses, en général si bien informées, si riches de citations et de renvois bibliographiques.

Le fait que l'auteur ait reçu sa formation dans les études d'histoire ancienne produit l'effet sans aucun doute positif d'aborder ces textes chrétiens avec la sensibilité particulière d'une personne attentive aux aspects de la vie quotidienne, sociale, économique et culturelle du monde gréco-romain. On y sent l'influence, reconnue et appréciée, de l'œuvre de Peter Brown, Paul Veyne et de beaucoup d'autres savants européens qui se sont mesurés à ces textes pleins de vitalité. Mais il est encore plus intéressant de voir comment l'auteur, en tirant profit de sa longue expérience d'archéologue et de ses connaissances vastes et approfondies de la littérature secondaire, ne perd pas l'occasion d'illustrer le commentaire des textes littéraires grecs et latins avec des renvois abondants et suggestifs au matériel archéologique constitué essentiellement de mosaïques et de pièces d'orfèvrerie.

Les thèmes traités dans le volume sont variés : on passe de la présentation de la réaction paienne au christianisme à l'étude de l'assimilation de la culture paienne dans le Pédagogue de Clément d'Alexandrie; de l'analyse de la société gréco-romaine chez les auteurs chrétiens (Clément et Jérôme) à l'impact social et religieux du monachisme chrétien, pour conclure par des témoignages patristiques sur les problèmes économiques et sociaux du Bas Empire (en premier lieu Salvien de Marseille) et par une analyse des théories et des expériences démonologiques présentées dans certains écrits provenant des milieux monastiques.

On pourrait dire que l'intérêt central de ces essais est constitué par l'histoire sociale et culturelle de l'Antiquité Tardive et par le rôle de l'élément chrétien dans cette histoire longue de plusieurs siècles. L'intérêt pour l'histoire religieuse du monde grec païen n'est certes pas prééminent. Le lecteur de cette revue peut néanmoins y trouver quelques allusions à celle-ci qui sont loin d'être insignifiantes ou secondaires.

En effet, le premier chapitre est consacré au thème de la réaction païenne au christianisme, un argument qui prend de plus en plus de valeur dans les études d'histoire religieuse païenne de la fin de l'antiquité. Les chapitres sur Clément sont pleins de citations de poètes et de philosophes grecs, et l'analyse de la contre-culture monastique met inévitablement en évidence les composantes proprement religieuses du monde païen contre lesquelles le message chrétien réagit de façon polémique. Il s'agit de pages perspicaces, très intéressantes et toujours bien documentées. On doit seulement regretter l'absence de quelques titres qui auraient certainement enrichi cet apport et l'auraient placé dans une perspective 
plus vaste, par exemple les livres de R.L. Wilken, The Christians as the Romans saw Them (New Haven-London, 1984) et de N. Zeegers-Vander Vorst, Les citations des poètes grecs chez les apologistes chrétiens du II siècle (Louvain, 1972).

Dans une prochaine édition du volume, on pourra facilement corriger les coquilles qui gênent la lecture (par exemple Rufin d'Alexandrie au lieu de Rufin d'Aquilée à la page 281 !). Malheureusement, même l'auteur de ce compte rendu a été victime d'une faute typographique regrettable à la page 208 , note 17 : P. Franeo, au lieu de Pier Franco Beatrice! Mais cela, évidemment, n'enlève rien à la valeur du livre qui trouve sa place à juste titre parmi les meilleurs travaux publiés au cours de cette décennie sur l'histoire sociale et religieuse de l'Antiquité Tardive.

Pier Franco Beatrice (Università di Padova)

Gérard Freyburger et Laurent Pernot (éds), Du béros païen au saint chrétien. Actes du colloque organisé par le Centre d'Analyse des Rhétoriques Religieuses de l'Antiquité (C.A.R.R.A.), Strasbourg, 1-2 décembre 1995, Paris, Institut d'Études Augustiniennes, 1997. 1 vol. $16 \times 24,5 \mathrm{~cm}, 231$ p. (Série Antiquité, 154). ISBN : 2-85121-159-5.

Le sujet n'est pas neuf, mais il est d'une richesse inépuisable, et la perspective ici adoptée, celle de la rhétorique religieuse, contribue à en renouveler l'étude. Dans leur avant-propos, les deux éditeurs indiquent clairement les buts assignés au colloque, - l'enquête se veut "fondée sur les textes et conduite au plus près des textes ", - et ils en synthétisent les apports. La plupart des 18 communications présentées sont centrées sur l'évocation d'un personnage singulier, historique ou mythique, dont on s'applique, le cas échéant, à faire voir les vicissitudes qu'a connues son exemplarité; d'Aristide le Juste à saint Séverin du Norique, au temps des invasions barbares, défilent successivement héros grecs et romains, saints et saintes chrétiennes, avec une section intermédiaire où sont examinés plusieurs cas intéressants de contamination des deux modèles; trois contributions examinent certains traitements du thème à l'époque moderne. Dans une remarquable conférence d'ouverture : "Le héros et le saint», J.-Cl. Fredouille esquisse la problématique du sujet, s'attachant à montrer les variations que subissent les modèles au gré des évolutions historiques, les continuités possibles entre les figures du héros et du saint, mais aussi leur irréductibilité, due essentiellement au caractère théocentrique de la conception chrétienne de la destinée. Trois index soignés (auteurs anciens cités, noms de personnes et de lieu) facilitent la consultation de l'ouvrage.

Cet intéressant colloque inaugurait les activités du C.A.R.R.A., lequel annonce aussi, pour bientôt, la publication d'une bibliographie relative à la prière dans l'Antiquité.

André Motte

(Université de Liège)

Raffaelle Pettazzoni, I Misteri. Saggio di una teoria storico religiosa, Presentazione di Dario Sabbatucci, Cosenza, L. Giordano Editore, 1997. 1 vol. $15 \times 21,5 \mathrm{~cm}, 245$ p. (Biblioteca di Studi Religiosi). ISBN : 88-86919-05-0.

Seventy-five years after the first publication, this welcome re-issue provides an opportunity to assess an important text of the history of religions: I Misteri by Raffaele Pettazzoni, the indisputable father of Italian historico-religious studies and founder of a school which numbers among its most prestigious exponents 